\title{
RFID Modular System for the Internet of Things (IoT)
}

\author{
Jaroslav Kadlec*, Radek Kuchta, Radovan Novotný and Ondřej Čožík
}

Department of Microelectronics, Brno University of Technology, Technická 3058/10, 61600 Brno, The Czech Republic

\begin{abstract}
Our paper presents an approach of how RFID technology can be used to simplify operations and improve the effectiveness and efficiency of inventory management. The goal of our research is to design system architecture for identifying and monitoring movement of monitored items. The basic requirement is to create a modular system and application of this system for real hospital laundry management application. We analysed possible solutions and available technologies. We also analysed a specific use-case of laundry management process for designed laundry monitoring system based on the loT platform principles. The development is related to the hot topic of the Internet of Things and the utilization of RFID technology as a key technology for detection and identification of monitored objects.
\end{abstract}

Keywords: Auto-ID system; Identification; Internet of things; RFID; Tag

\section{Introduction}

This paper describes the main outcomes of our research which is focused on the problematic of design and development of a universal system for monitoring the movement of objects and things. In the second chapter of the paper there is an analysis and state of the art of similar solutions and available technologies which can be used in the specific case of laundry management in a hospital environment. An application scenario and detailed description of a use-case and operation during laundry circulation process is in chapter two. Based on collected requirements and system specification we developed a universal open system which can be applied to a wide range of application scenarios requiring monitoring the movement of things such as logistic applications and software for control and management of a whole range of items. The third chapter is focused on the explanation of a developed system topology and its main properties and functions followed by the description of developed tools and modules for system management and testing. The last chapter summarizes our achievements and discusses the next steps of how we will continue in our following research.

\section{State of the Art and Importance of RFID Auto-ID System for the Internet of Things}

Preparation work for our research consisted of collecting and analysing documents concerning available concepts of IoT systems, used identification technologies and identification tags. The main results of this preparatory phase were published in our journal paper describing state of the art of identification technologies [1].

\section{Internet of Things}

The term the Internet of Things (or IoT for short) was proposed by Kevin Ashton in 2009 [2] and the concept became popular through the Auto-ID Centre at MIT [3]. A thing can be a physical object with a virtual representation in a network Internet-like structure. It is the network of physical objects accessed through the Internet, that are equipped with unique identifiers and an ability to automatically transfer data through a network without requiring manual data entry.

The IoT network infrastructure with self-configuring capabilities interconnects various places, such as manufacturing or healthcare facilities, warehouses, place of supply of services, energy grids, and logistics systems, to the Internet. Such an information network based on standard and interoperable communication protocols is formed by objects having identities, operating in smart spaces using intelligent communication interfaces. This type of information network represents a critical part of the connectivity platforms for system control, and provides opportunities to improve processes, and significantly reduce risks.

Radio Frequency Identification (RFID) as the technology for IoT can be used to identify and track items [4-6]. It is a perfect identification technology for our development purposes due to its typical parameters such as wireless reading in the range of several meters, fast reading of numerous identification tags at one time and the possibility to have passive tags without an internal source of energy [6]. RFID is the newest identification technique among all IoT technologies $[7,8]$. When an object can represent itself digitally, it can be controlled more precisely from elsewhere with high efficiency. If objects are equipped by identifiers, they can be managed and inventoried automatically; therefore, it is then possible to track the full transportation path of these products. When objects can both sense the operating environment and communicate, they become agents for understanding of real-time events.

\section{The RFID Auto-ID system and RFID based identification technologies}

The RFID systems may be considered a case of a wider class of Automatic Identification (Auto-ID) systems [9], i.e. RFID AutoID System [10]. In the IoT, objects are expected to become active participants in business, information and control processes. For example an RFID laundry tag [11] is capable of withstanding washing conditions when attached to linen and it can be used to track its usage. It will offer specific object identification, and connection capability as the basis for development of independent cooperative services and applications. In the IoT system available services are able to interact with "smart" objects, query and change their state and collect associated information. As the new networks link data from products,

*Corresponding author: Jaroslav Kadlec, Department of Microelectronics, Brno University of Technology, Technická 3058/10, 61600 Brno, the Czech Republic, Tel: 420541145 111; E-mail: kadlecja@feec.vutbr.cz

Received April 24, 2014; Accepted June 27, 2014; Published July 03, 2014

Citation: Kadlec J, Kuchta R, Novotný R, Čožík O (2014) RFID Modular System for the Internet of Things (IoT). Ind Eng Manage 3: 134. doi: 10.4172/21690316.1000134

Copyright: $\odot 2014$ Kadlec J, et al. This is an open-access article distributed under the terms of the Creative Commons Attribution License, which permits unrestricted use, distribution, and reproduction in any medium, provided the original author and source are credited. 
physical assets, or the operating environment, they will generate better information and analysis, which can significantly enhance decision making process. Companies can begin to track and count everything, and greatly reduce waste, loss and cost - the range of possible uses for tracking is expanding [5]. The major advantage of using RFID tags is that multiple RFID tags can be read at the same time, and they can be hidden inside materials and objects, unlike barcodes which can only be read one at a time and need to be placed outside on items to be scanned.

Fuhrer et al. [12] show how technologies of identification by RFID can be used to build a smart hospital which optimizes processes, and enhances the quality of service. This paper also presents how the technology can be used for an assets tracking application. Thanks to several initiatives several open-source RFID frameworks, such as foosstrak project [13], Aspire RFID [14] and Mobitec [15] have been developed which provide unlicensed use of RFID middleware stacks. These open source RFID frameworks contain numerous modules and tools and require deep knowledge of their internal principles and mechanism together with a considerable amount of development work to install or modify these solutions for the specific conditions of a real application. Schwartz [16] describes Microsoft's commercial approach to RFID that offers a way of communication and control of RFID devices on the Microsoft Windows platform. This solution is called BizTalk RFID and includes software layers approach - ones that allow all types of RFID devices, sensors, and EPC (Electronic Product Code) readers to be integrated in a plug-and-play method. Other commercial platforms for RFID are Rifidi [17], Aspire RFID [18], Oracle, IBM, Detego [19], OAT RFID middleware platform [20] and DEPCAS [21]. In comparison with the previously mentioned RFID platforms our system has similar functionality, however, it adds one unique function which is the evaluation of the direction of an identification tag's passage and provides a simpler system configuration and management but it is not primarily designed for large installations with numerous facilities in different locations or countries. Our platform is primarily targeted to smaller installations such as, for example, a hospital laundry management system whose use-case is presented in the following chapter.

\section{RFID Laundry Inventory System}

This paper also describes a case study of how RFID technology can be used to simplify operations and improve the effectiveness and efficiency of laundry management. This use-case gives a better understanding of the main advantages of the proposed platform and shows the benefits of its implementation to a real application environment. Since the laundry RFID tags can be attached to cloth and linen, the possibility of reading laundry information has raised opportunities for automatic identification. The IoT technology is opening new frontiers for improving and changing processes and consumer interactions and even entire business models can be fine-tuned to take advantage of accessible data. It will have implications for organizational-structures, as well as for the way of how decisions are made, operations are managed, and for optimization of processes in which traditional approaches have not brought satisfactory results. Changes in information patterns have created a market for entirely new solutions and more innovative ways to enable new services, smarter decisions and business models, and reduce costs.

\section{Use-cases related to the requirements of a laundry management system and managerial implication}

For example, a manual laundry tracking process is very labour intensive, because obtaining detailed records requires it to separate, count, record (e.g. by handwriting notes) and insert into a computer different types of laundry items [22]. Cost effective and efficient laundry management demands sophisticated laundry management software and tagging systems. An advanced automatic identification technology based on the RFID technology would have significant added value for inventory systems (i.e. computer data can in real-time show if the inventory stored in the stock is correct and has adequate quantity).This solution with many managerial implications can reduce the time and effort necessary for processing, shipping and receiving the laundry, and provide ultimate control and accountability in the process of laundry circulation - (e.g. status, current location, last recorded time, number of left washing cycles, information about used material and washing conditions, etc.). The basic infrastructure necessary for this type of practical application includes readers and tags, as well as RFID management software, all designed to work together seamlessly.

It was necessary to map the processes to refine the system requirements. The requirements for locating RFID readers for comprehensive coverage were identified by mapping the movement of monitored laundry items. Based on the results of mapping a process the possible readers location and requirements for the RFID readers, and modes of operation was determined. The system allows four basic modes, namely:

- Simple reporting, single report mode - the first occurrence of the monitored items is reported. As long as the item leaves the signal range of the antenna for a specified period of time, there is no further reporting.

- Simple reporting by each antenna- similar to simple reporting, but associated with a single antenna. If the laundry is moved within the second antenna signal range, it is also reported by the other antenna.

- Periodic reporting - if the laundry is within range of the antenna, there is a periodic report from the antennas with the strongest signal.

- Detection of passage direction - in the case of two, or a multi - port reader, passage direction is determined on the basis of signals from the antennas. For example, if the first antenna is considered external antenna and a second antenna for the internal antenna, the passage between 1 and 2 is reported as the direction of "IN" and the passage between 1 and 2 is reported as "OUT".

All of these modes can be used on any connected reader. Algorithms for evaluating signals from readers are implemented at the middleware level.The modularity of the solution then lies in the possibility of adding another RFID reader. This reader can be operated in any of the four modes described above. The information gained from the report of the readers and antennas can thus by used for specific use applications.

A general schematic of laundry circulation in a hospital environment is in Figure 1. All operations and steps which follow the movement of linen from the wash house to user and back are illustrated. Linen can be monitored after or during all these steps and the system operator has real-time information about the actual position of single linen item, but also about its general condition (e.g. number of completed washing cycles and its condition), total amounts, losses and damages, and can immediately react to new linen requests. The RFID IoT system can be also helpful in processes where the operator needs to be informed about i.e. an incorrect washing program or maximum temperature of ironing of the processed linen, because all this information is stored, managed 
Citation: Kadlec J, Kuchta R, Novotný R, Čožik O (2014) RFID Modular System for the Internet of Things (loT). Ind Eng Manage 3: 134. doi: 10.4172/2169-0316.1000134

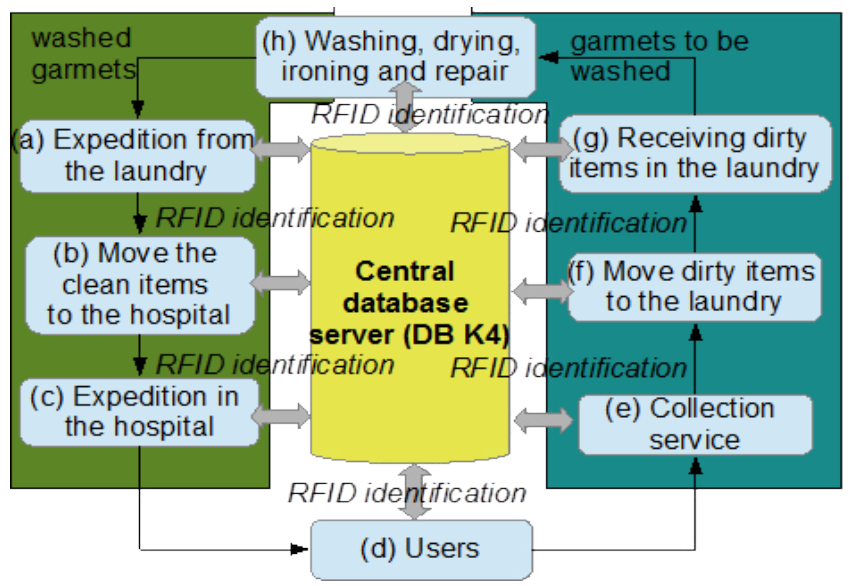

Figure 1: A process diagram of the hospital laundry circulation: overview for use cases documentation.

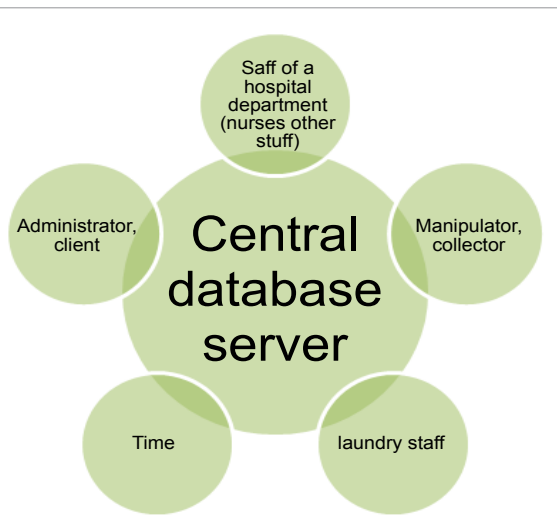

Figure 2: Actors for hospital laundry circulation use-case initialization

and can be provided by the RFID IoT system for laundry management. Areal example of processes which correspond to a laundry management system are summarized in Table 1 and all included parts of the hospital laundry circulation use-case are shown in Figure 2. Based on a detailed analysis of all operations during the laundry circulation process we identified the best points for placement of the RFID readers and their antennas which would ideally cover the complete process of laundry circulation. There are two modes of monitoring movement of laundry items. Firstly, passage detection mode which requires only one antenna. Secondly, there is possibility of monitoring movement of items using multi - antenna readers.

\section{Realization and Description of Necessary Network Infrastructure}

We analysed possible solutions of a communication layer for data transfer between different types of RFID readers and a master system device. Based on our research of available RFID readers technology we chose Ethernet as a standardized communication interface which allows us to connect most of the readers on the market to our system [23].

We designed a middleware layer which combines a data communication part with a data processing core and provides preprocessed data to an upper system layer. This layer structure with an Ethernet communication transport interface makes this solution universal for wide usage across different identification technologies and provides unified data to upper systems which will implement this middleware. The proposed network infrastructure, Figure 3, can be used in all types of companies and applications because it can use existing network infrastructure. Realization of the designed middleware layer was done in the Microsoft .NET 4.0 framework to have a platform independent and Microsoft Windows OS compatible solution.

One of the main goals of our development work was to realize a complex SW module for obtaining data from readers, filtering, unifying and providing these data for following evaluation or storing into a database system in an external database server [6]. For filtering and

\begin{tabular}{|c|c|c|}
\hline Processes & Realized steps & Roles \\
\hline $\begin{array}{l}\text { (a) Expedition } \\
\text { from the laundry }\end{array}$ & $\begin{array}{l}\text { a1. Linen is categorized and packed } \\
\text { according to its final destination. } \\
\text { a2. Preparation for delivery to the } \\
\text { specified destination. }\end{array}$ & $\begin{array}{l}\text { Laundry workers } \\
\text { give over } \\
\text { Operator proceeds }\end{array}$ \\
\hline $\begin{array}{l}\text { (b) } \\
\text { Move the clean } \\
\text { items to the } \\
\text { hospital }\end{array}$ & $\begin{array}{l}\text { b1. Delivery of clean linen is every working } \\
\text { day. The pavilion is typically operated with } \\
\text { a car transporting the bags of dirty linen. } \\
\text { Alternatively, it is possible to use trucks. }\end{array}$ & Operator moves \\
\hline $\begin{array}{l}\text { (c) } \\
\text { Expedition in the } \\
\text { hospital }\end{array}$ & $\begin{array}{l}\text { c1. Hospital takes the clean linen. } \\
\text { c2. The linen is taken and shipped to the } \\
\text { specified destination. }\end{array}$ & $\begin{array}{l}\text { Operator gives } \\
\text { over } \\
\text { Medical orderly } \\
\text { takes and sorts }\end{array}$ \\
\hline $\begin{array}{l}\text { (d) } \\
\text { Users } \\
\text { (circulation within } \\
\text { the department) }\end{array}$ & $\begin{array}{l}\text { d1. The clean linen is placed in a cabinet } \\
\text { or rack according to the type of packaging. } \\
\text { d2. Washed or newly added pieces are } \\
\text { stored. } \\
\text { d3. Personal laundry after washing is } \\
\text { returned directly to the user. } \\
\text { d4. Using hospital laundry inside the } \\
\text { department, bed linen can be transferred } \\
\text { to patients. } \\
\text { d5. Collection of dirty laundry. Dirty } \\
\text { laundry is collected according to type and } \\
\text { placed in bags, prepared to move to the } \\
\text { laundry room. }\end{array}$ & $\begin{array}{l}\text { Hospital personnel } \\
\text { use } \\
\text { Hospital personnel } \\
\text { collect } \\
\text { Medical orderly } \\
\text { takes away }\end{array}$ \\
\hline $\begin{array}{l}\text { (e) Collection } \\
\text { service } \\
\text { (collecting place) }\end{array}$ & $\begin{array}{l}\text { e1. From collection points bags of dirty } \\
\text { laundry are taken away periodically } \\
\text { (typically once a day). The trucks moved } \\
\text { the dirty linen to the centralized collection } \\
\text { point before leaving. } \\
\text { e2. Evidence of bags, identification data } \\
\text { and requisitions for information, what } \\
\text { clothes will be delivered (quantity and } \\
\text { type) and where. Dirty laundry is not } \\
\text { counted. }\end{array}$ & $\begin{array}{l}\text { Medical orderly } \\
\text { gives over } \\
\text { Operator takes } \\
\text { away }\end{array}$ \\
\hline $\begin{array}{l}\text { (f) } \\
\text { Move dirty items } \\
\text { to the laundry }\end{array}$ & $\begin{array}{l}\text { f1. The pavilion is typically operated with a } \\
\text { car that transports the bags. An alternative } \\
\text { way of moving for shorter distances may } \\
\text { be to use a wheelchair. }\end{array}$ & Operator moves \\
\hline $\begin{array}{l}\text { (g) Receiving } \\
\text { dirty items in the } \\
\text { laundry }\end{array}$ & $\begin{array}{l}\text { g1. Sort the laundry by washing programs, } \\
\text { or by type. } \\
\text { g2. Linen from operating rooms and } \\
\text { infectious linen is always in separate } \\
\text { bags. Infectious clothes are washed } \\
\text { separately in the "soluble" bag. }\end{array}$ & $\begin{array}{l}\text { Operator gives } \\
\text { over } \\
\text { Laundry workers } \\
\text { takes away }\end{array}$ \\
\hline $\begin{array}{l}\text { (h) Washing, } \\
\text { drying, ironing } \\
\text { and repair }\end{array}$ & $\begin{array}{l}\text { Processes for handling of dirty laundry: } \\
\text { h1. Laundry, divided into categories, is } \\
\text { moved to the wash. } \\
\text { h2. The next step is washing and other } \\
\text { processes. Poorly washed pieces are } \\
\text { washed again. } \\
\text { Processes for handling of clean laundry: } \\
\text { h4. Sewing and repair of minor damage. } \\
\text { Items which cannot be repaired are } \\
\text { postponed for decommissioning. } \\
\text { h5. Hand sorting of laundry by name and } \\
\text { department. }\end{array}$ & $\begin{array}{l}\text { Laundry workers } \\
\text { wash laundry }\end{array}$ \\
\hline
\end{tabular}

Table 1: Typical processes associated with the circulation of hospital laundry. 
Citation: Kadlec J, Kuchta R, Novotný R, Čožik O (2014) RFID Modular System for the Internet of Things (loT). Ind Eng Manage 3: 134. doi: 10.4172/2169-0316.1000134

Page 4 of 7

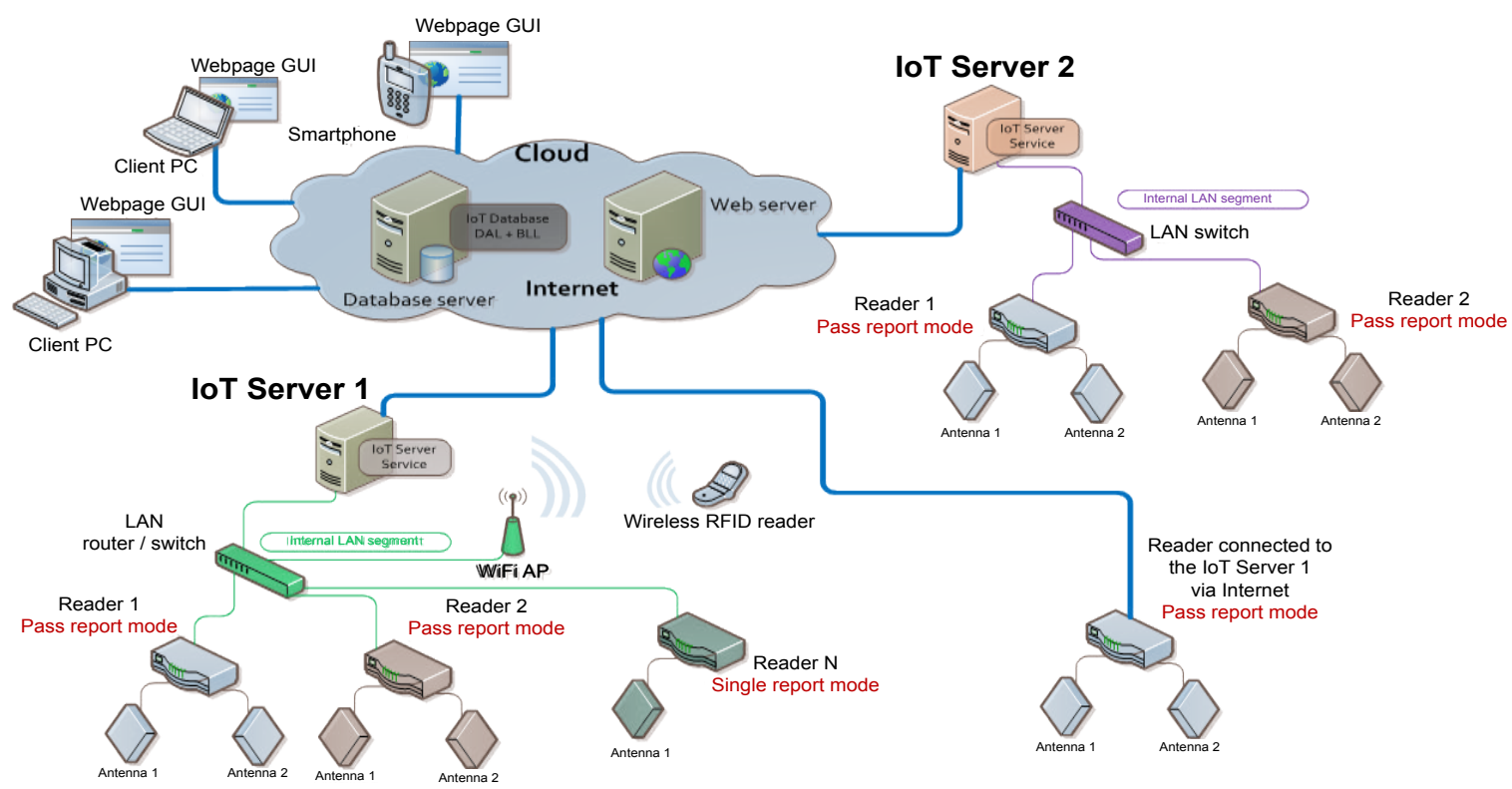

Figure 3: Proposed network infrastructure of the developed loT system.

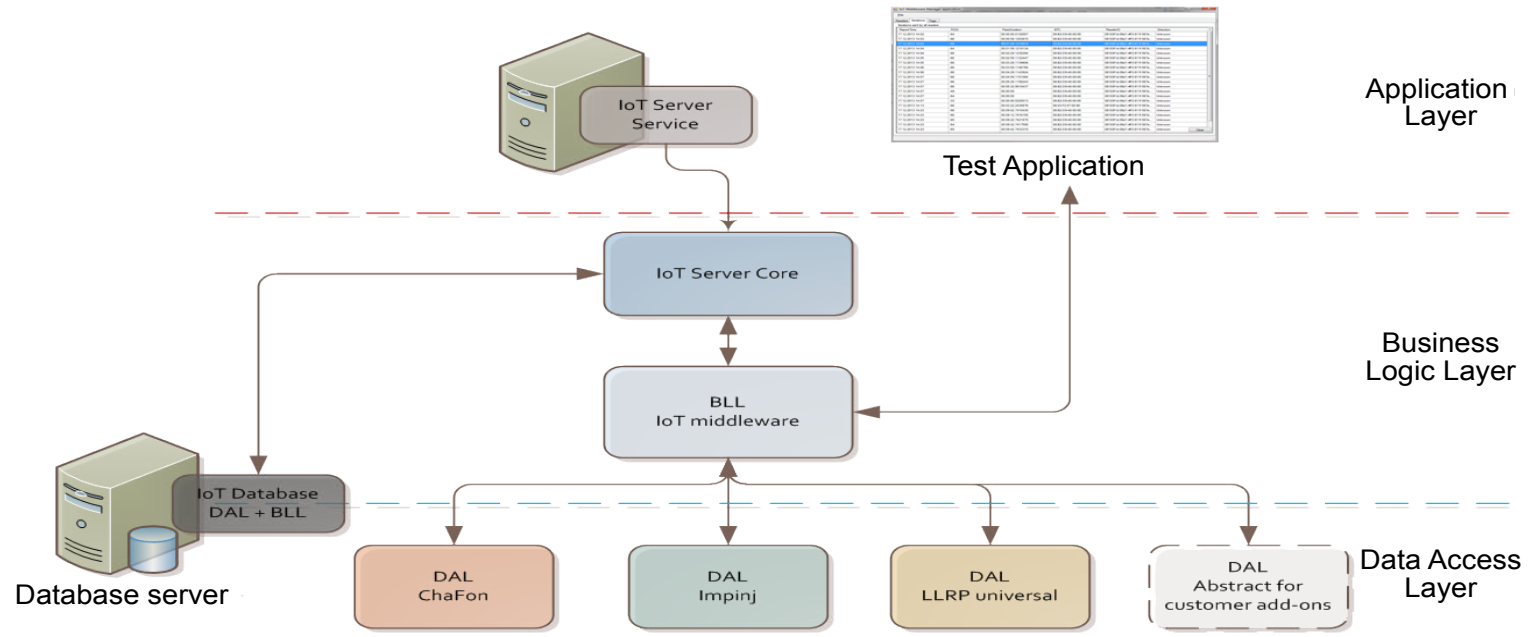

Figure 4: Layer diagram of the developed system for data acquisition and filtering of the UHF readers identification tags information.

unifying purposes the IoT middleware has to control reading processes of all connected readers and process the raw data from the antennas which contains information only about tags in the communication range of an antenna and some of the readers add the RSSI information. Collected data are analysed, filtered and processed by the algorithm for determining the direction of passage. This information is then transferred to a superior system in the form of a single iteration report. The lowest level of the developed SW module is a communication layer which provides several communication protocols according to required protocols by different readers from different manufacturers. This communication layer is made universal and developers can freely add other communication protocols via the available SW interface. Currently, there are two implemented protocols. The first one is standardized LLRP (Low Level Reader Protocol) which is supported by major manufacturers of top class RFID readers and the second one is a proprietary protocol for a specific manufacturer: ChaFon, which offers low-cost RFID readers. The LLRP determines a protocol which provides access to air protocol command parameters and arranges a control of RFID air protocol operating timing [3].

The LLRP processes data from every single RFID tag in the form of EPC (Electronic Product Code) and a configuration of the reader (e.g. settings of reading intervals, transmit power, sensitivity of antennas etc.).

The advantage of this approach is the possibility of using an LLRP reader with the support of different manufacturers and thus creating a standardized interface depending on the model EPCIS (Electronic Product Code Information System). The EPCIS is an EPC global standard that specifies a means to enable different applications to leverage EPC data via EPC-related data sharing [17]. The structure of the developed IoT middleware that processes data obtained from the connected readers can be seen in Figure 4. 
Citation: Kadlec J, Kuchta R, Novotný R, Čožik O (2014) RFID Modular System for the Internet of Things (loT). Ind Eng Manage 3: 134. doi: 10.4172/2169-0316.1000134

Page 5 of 7

The next goal of our development was to provide secure communication and remote reader configuration and upgrades via a secure channel. This was ensured by several security mechanisms at a communication and application level. Upgrade of FW in readers is also possible due to the modular structure of IoT middleware which conveys FW data transport to the reader. Its installation and calling system commands is done by DAL (Data Access Layer) of the relevant reader. The data access layer provides a way to give access between the program and any type of persistent storage. To ensure the versatility of the proposed solution, the configuration data is passed to the data link layer in XML format. A data string in XML format is decoded on the lowest layer and the relevant parameters of the reader are then set.
This solution allows simple management of the settings of each reader, upgrading to the newer FW versions and storing these settings in a database system. Implementation of a non-standard communication protocol shows the main advantage of this modular concept, which allows easy system expansion and addition of other types of readers from different manufacturers into the system.

Another part of the developed software is a database system that allows archiving data from the readers. This database is used for longterm testing. Data from individual transactions and iterations of tags are stored and used for searching of error states and their elimination. Relational database structure is shown in Figure 5. The database is

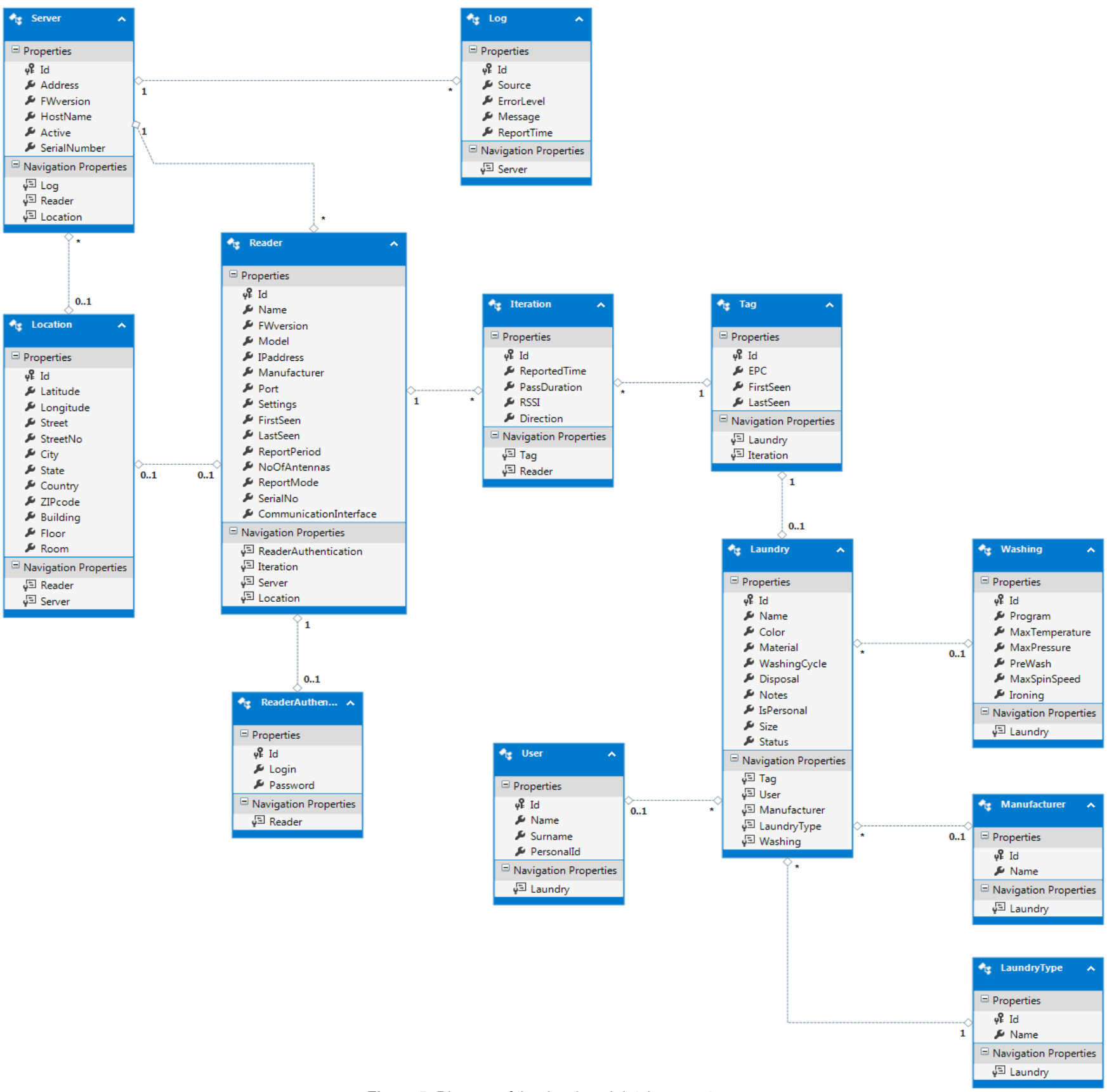

Figure 5: Diagram of the developed database system. 
organized into several blocks which make it universal for usage not only in laundry management but also for other types of IoT monitoring systems. Core database tables store information about IoT system structure and processed tags and the rest of the database tables is specific for laundry management. The mutual connection of these two parts provides an identification number of a tag. Thus, every tag can be linked via its ID to other databases primarily targeted to storage data from different monitoring processes or application scenarios.

For demonstration, testing and verification of IoT middleware a SW tool was realized which controls various connected RFID readers and displays information about recorded iterations of tags with the connected readers. Testing the SW tool allows easy configuration of the settings of every connected reader and real-time monitoring of their status via the implemented IoT middleware library. A developer or system administrator can have access to real-time system data with processed information about iterations of tags within detection range of readers' antennas. This makes the necessary development feedback about internal processes and general functionality of the system. The realized testing SW tool makes development of filtration algorithms easier with the possibility to have immediate debugging data output and simplifies the configuration of the readers.

The user is immediately informed about the response of the system to individual passages of UHF tags through the detecting field of the RFID antennas of individual readers.

This information provides the necessary feedback for the development of algorithms for determining the direction of passage of the UHF identification tags, but also for the settings parameters (e.g. gain and sensitivity of the antenna) and finding optimal physical placement of antennas

\section{Conclusions}

Our paper presents an approach of how RFID technology can be used to simplify operations and improve the effectiveness and efficiency of inventory management [24-28]. The main goal of our research was fulfilled by the development and realization of a universal and freely extensible system for wireless monitoring of things and objects. We have found that by using one four-port RFID reader we can monitor four laundry operations, thereby reducing installation costs. In relation to the state of the art could be said that the presented network topology creates a universal platform for access to data from different RFID readers from different vendors which have different communication protocols and standard Implemented in them. Also, part of our research was focused on an evaluation of reliable and fast algorithms for determining the direction of passage of the identification tag by the field of connected antennas from data from all types of RFID readers. This function that give the information if laundry is going in or out is unique and it is not implemented in other RFID management systems which were mentioned in chapter 2.2. Other research effort was spent on development of a module for unifying data from the RFID readers. Due to different communication protocols, data formats, and different ways of obtaining data from RFID readers. These are moreover dependent on the applied settings. The unified module had to be developed and implemented as a data pre-processing part of the IoT middleware.

In the first step of our research we analysed possible solutions and available technologies applicable for the designed IoT identification system. Based on state of the art of identification technologies we chose the UHF RFIDtechnology, which fulfils all important parameters and can be used as a universal monitoring platform. We also analysed a specific use-case of laundry management process in a hospital and from the description of process operations we found and specified in detail all the important requirements to the designed laundry monitoring system based on the IoT platform principles.

Based on the specification of processes, data flows and network topology we designed a layered structure of the IoT system. The core part of this system is the IoT middleware which is responsible for processing data from the lowest system architectural layers. The developed system is module based and can be extended by add-ons for communication with other types of RFID readers. The basic condition for development of a new add-on module is to implement a standardized interface for data transmission. Currently there are three developed communication addon modules available for demonstration of implementation principles and usage purposes of a real application. The first add-on module is for communication via the proprietary communication protocol with an RFID reader from the manufacturer ChaFon. The second one is for communication with an Impinj reader through the LLRP protocol with some extended commands and the third add-on module is for a general device which supports the LLRP communication protocol standard.

The IoT middleware compiles data from all connected RFID readers, filters them and according to the selected reading mode of every single RFID reader it applies an algorithm for iteration evaluation. The resulting iteration reports contain all important parameters, about time, place, average signal strength, duration and direction of passage of a tag, and information about the identification tag itself which is initiator of this transaction. Collected iterations by the IoT middleware are then reported to the upper system layer for post-processing operations and storage in a database system.

The realized database system is a cloud based solution which offers unlimited access to data from anywhere where users can be connected to the Internet. The user can obtain all information from the laundry management system anywhere s/he needs it.

Our future work will be focused on the realization of a web server application which will provide a Web based Graphical User Interface for user-friendly control and management of a whole realized IoT system via the Internet, not only for classical PCs but also for smartphones and tablets.

\section{Acknowledgements}

The research presented in this paper is funded by the Ministry of Industry and Trade of the Czech Republic within the framework of research projects no. FR-TI4/530, FR-TI1/057 and by the research project CEITEC - Central European Institute of Technology CZ.1.05/1.1.00/02.0068.7.

\section{References}

1. Kadlec J, Kuchta R, Novotný R (2013) Overview of identification technologies for the Internet of Things concept network between various objects 69:114-20.

2. MIT Auto-ID Labs launches Cloud of Things initiative (2013)11-10

3. Ashton K. (2013-10-14) That 'Internet of Things' Thing. RFID Journal LLC.

4. Ahson S, llyas M ( 2008) Applications, Technology, Security, and Privacy RFID: Boca Raton: CRC Press.

5. Bartneck N, Klaas V, Schönherr H .( 2009) Optimizing Processes with RFID and Auto ID. Erlangen: Publicis Publishing.

6. Roussos G (2008) Networked RFID: Systems, Software and Services. London: Springer - Verlag.

7. Sikander J. (2014-02-20) Microsoft RFID Technology Overview.

8. Lehpamer H.( 2012) RFID Design Principles:Artech House Norwooe.

9. Bidgoli H.(2006) Handbook of Information Security, Threats, Vulnerabilities, Prevention, Detection, and Management:John Wiley \& Sons, New Jersey.

10. Radio Frequency Identification (RFID) Systems(2013-10-17). 
Citation: Kadlec J, Kuchta R, Novotný R, Čožik O (2014) RFID Modular System for the Internet of Things (loT). Ind Eng Manage 3: 134. doi: 10.4172/2169-0316.1000134

11. BACHELDOR B.( 2013-03-07) RFID News Roundup.

12. Fuhrer P, Guinard D( 2014-02-27) Building a Smart Hospital using RFID technologies.

13. Open Source RFID Platform( 2014).

14. ASPIRE for SME(2014).

15. CUHK EPC Global RFID Middleware 1.0 (Open Source) (2014).

16. Schwartz K (2014) BizTalk RFID: Making RFID Deployments Easy, Simple and Economical.

17. Rifidi - Connect the Internet of Things.(2013).

18. AspireRFID Middleware.(2013)

19. Detego® Integration Platforms( 2013).

20. OAT Technology( 2013).

21. Cerrada C, Abad I, Cerrada J, Depcas(2010) An industrial approach to RFID middleware In Industrial Technology (ICIT), 2010 IEEE International Conference, $1394-1399$.
22. Laico K ( 2004) Laundry and Linen Services in Hospitals. E-journal of AECS illumination 21-25.

23. Hunt D, Puglia A, Puglia M (2007) RFID: A Guide to Radio Frequency Identification: John Wiley \& Sons: New Jersey.

24. Zhang Y ( 2012) RFID-enabled real-time manufacturing information tracking infrastructure for extended enterprises. Journal of Intelligent Manufacturing, 2357-2366.

25. Stephan P ( 2013) Applying digital product memories in industrial production. In: SemProM, 283-304 Berlin Heidelberg.

26. Kelepouris T, Mcfarlane D, Giannikas V ( 2012) A supply chain tracking model using auto-ID observations International Journal of Information Systems and Supply Chain Manag1ement (IJISSCM) 5: 1-22.

27. Jia X (2012) RFID technology and its applications in Internet of Things (IoT). In: Consumer Electronics, Communications and Networks1285.

28. Kelepouris T, Mcfarlane, D, Giannikas V (2012) A supply chain tracking model using auto-ID observations. International Journal of Information Systems and Supply Chain Management (IJISSCM) 5: 1-22. 